INTERVENTIONAL CARDIOLOGY AND SURGERY

\title{
Prognostic role of cardiac troponin I after percutaneous coronary intervention in stable coronary disease
}

\author{
T Nageh, R A Sherwood, B M Harris, M R Thomas
}

Heart 2005;91:1181-1185. doi: 10.1136/hrt.2004.042911

See end of article for authors' affiliations

Correspondence to: Dr Thuraia Nageh, Department of Cardiology, King's College Hospital, Denmark Hill, London SE5 9RS, UK; tnageh@hotmail. com

Accepted 23 August 2004

\begin{abstract}
Objective: To assess the role of cardiac troponin I (cTnl) in predicting outcome after percutaneous coronary intervention (PCI).

Methods and results: cTnl was measured immediately before and at 6, 14, and 24 hours after $\mathrm{PCl}$ in 316 consecutive patients with stable symptoms and native coronary artery disease. The study end point was the occurrence of major adverse cardiac events (MACE) at 30 days and at 18 months after PCl: death, $Q$ wave myocardial infarction (MI), or repeat revascularisation in hospital. Postprocedural cTnl increased in $31 \%$ of patients. The cumulative MACE rate at 18 months was $25 \%$ ( $17.7 \%$ due to repeat $\mathrm{PCl}$ procedures). There was a significant association between postprocedural cTnl increase and death, $\mathrm{Q}$ wave $\mathrm{Ml}$, or both (odds ratio (OR) 3.28, 95\% confidence interval $(\mathrm{Cl}) 1.7$ to $6.4, \mathrm{p}=0.01$ ). Post- $\mathrm{PCl}$ cTnl increase had a positive predictive value (PPV) for adverse events at 18 months of 0.47 and a negative predictive value (NPV) of 0.96 (OR 18.9, 95\% Cl 9.7 to $37, \mathrm{p}<0.0001$ ). The presence of both a postprocedural cTnl rise and a procedural angiographic complication gave a PPV for adverse events of 0.69 and an NPV of 0.92 (OR 22.6, 95\% Cl 2.6 to 68.6, $\mathrm{p}=0.0005$ ).

Conclusions: $\mathrm{cTnl}$ increased post-procedurally in one third of this stable patient population undergoing elective $\mathrm{PCl}$ and was independently and significantly predictive of an increased risk of adverse events at 18 months, predominantly in the form of repeat $\mathrm{PCl}$.
\end{abstract}

$P$ ostprocedural creatine kinase and its MB isoform reportedly increase asymptomatically in as many as $26 \%$ of patients undergoing otherwise uncomplicated percutaneous coronary intervention (PCI), with an associated increase in adverse events in the short and long term. ${ }^{1-3}$ The cardiac troponins $\mathrm{T}$ and $\mathrm{I}$ (cTnI) are highly sensitive and specific markers of myocardial cell injury and necrosis. ${ }^{45}$ In addition to the strong diagnostic role of cardiac troponins, their prognostic value has become increasingly well established for patients presenting with acute coronary syndromes. ${ }^{6-10}$ However, there have been conflicting reports on the value of troponins in the setting of PCI in stable and unstable coronary disease. ${ }^{11-15}$ In this study, we evaluated the short and long term prognostic value of cTnI in patients presenting with stable, native coronary disease and undergoing elective PCI.

\section{METHODS}

\section{Study population}

We prospectively studied patients presenting to our institution between June 1999 and October 2000 with symptoms of stable angina ${ }^{16}$ or functional evidence of silent ischaemia in the presence of medical treatment. All patients had angiographic native coronary disease deemed suitable for PCI according to current American College of Cardiology (ACC)/ American Heart Association (AHA) guidelines. ${ }^{16}$ The study fully complied with the Declaration of Helsinki and was approved by our local research ethics committee. All patients gave their written, informed consent.

\section{Blood sampling and specimen analysis}

Blood was sampled for cTnI into tubes without anticoagulant immediately before and at 6,14 , and 24 hours after PCI. The samples were centrifuged at $2000 \mathrm{~g}$ for 10 minutes and the serum was separated and stored at $-20^{\circ} \mathrm{C}$ before analysis. cTnI was analysed quantitatively with the Bayer Immuno- 1 assay, a heterogeneous sandwich magnetic separation system consisting of mouse monoclonal and goat polyclonal cTnI antibodies. The detection antibodies were labelled with alkaline phosphatase and addition of substrate subsequently resulted in a colour change directly proportional to the analyte concentration. The assay had a detection limit of $0.1 \mu \mathrm{g} / \mathrm{l}$ and an analytical range up to $200 \mu \mathrm{g} / \mathrm{l}$. The interassay coefficient of variation was $6 \%$ at a concentration of $0.2 \mu \mathrm{g} / \mathrm{l}$, which was the manufacturer's recommended clinical discriminant value for significant myocardial injury and our chosen cut off value for defining troponin positivity.

\section{Interventional procedures}

PCI (balloon angioplasty, stent insertion, and rotational atherectomy) was carried out according to established practice at our institution, through 6 French femoral arterial sheaths and with 2-5000 units (weight adjusted) of heparin administered at the outset. All the patients were established on aspirin before PCI. All patients receiving coronary stents were given a $300 \mathrm{mg}$ loading dose of clopidogrel and $75 \mathrm{mg} /$ day for four weeks after the procedure.

\section{Study end points and patient follow up}

The study end point was the occurrence of major adverse cardiac events (MACE) in hospital, at 30 days, and at 18 months' follow up. MACE were defined as cardiac death, Q wave myocardial infarction (MI), repeat PCI, or coronary artery bypass grafting (CABG). Q wave MI was diagnosed in the presence of clinical symptoms, new significant $(\geqslant 1 \mathrm{~mm}$ depth) Q waves in at least two contiguous ECG leads, and a creatine kinase-MB concentration $>99$ th centile of our own

Abbreviations: ACC, American College of Cardiology; AHA, American Heart Association; CABG, coronary artery bypass grafting; $\mathrm{Cl}$, confidence interval; cTnl, cardiac troponin I; CURE, clopidogrel in unstable angina to prevent recurrent events; $M A C E$, major adverse cardiac events; $\mathrm{Ml}$, myocardial infarction; NPV, negative predictive value; $\mathrm{OR}$, odds ratio; $\mathrm{PCl}$, percutaneous coronary intervention; PPV, positive predictive value 
laboratories' reference control value. Cardiac troponins did not form part of our definition, as cTnI was being specifically assessed as a variable in this study.

Patients were followed up for the study end points by direct clinical review, telephone interviews, hospital medical records, and information from the referring physicians. Patients were not subjected to further coronary angiography unless it was clinically indicated.

\section{Statistical analysis}

Continuous variables were expressed as mean (SD). Noncontinuous (skewed) data were expressed as medians (interquartile range). Discrete variables were expressed as counts and percentages. The Student's $t$ test was used for comparison of continuous variables and Pearson's $\chi^{2}$ analysis was used to compare categorical variables. Patients' periprocedural clinical, angiographic, and procedural variables were subjected to a stepwise univariate analysis of possible predictive variables for postprocedural cTnI increase with the Cox proportional hazards regression model. The same model was used to identify variables that had a significant association with postprocedural adverse events. All variables with a significant univariate association with either postprocedural cTnI increase or adverse events at follow up were included in a multivariate proportional odds model and subjected to further analysis. $\chi^{2}$ analysis was also used to derive the positive (PPV) and negative predictive values (NPV) of procedural angiographic complications and postprocedural cTnI increase for clinical outcome. Kaplan-Meier survival analysis was applied to the postprocedural troponin

Table 1 Patient demographic, clinical, and baseline angiographic data

\begin{tabular}{|c|c|}
\hline Number of patients & 316 \\
\hline Number of vessels & 427 \\
\hline Number of men & $224(71 \%)$ \\
\hline Age (years)* & $58.6(6)$ \\
\hline Number aged $>70$ years & $97(31 \%)$ \\
\hline \multicolumn{2}{|l|}{ Risk factors } \\
\hline Smoking & $222(70 \%)$ \\
\hline Diabetes & $56(18 \%)$ \\
\hline Hypercholesterolaemia & $199(63 \%)$ \\
\hline Hypertension & $145(46 \%)$ \\
\hline Family history & $153(48 \%)$ \\
\hline \multicolumn{2}{|l|}{ CCS functional class } \\
\hline I & $16(5 \%)$ \\
\hline ॥ & $155(49 \%)$ \\
\hline III & $145(46 \%)$ \\
\hline IV & 0 \\
\hline \multicolumn{2}{|l|}{ Drug treatment } \\
\hline Aspirin & $310(98 \%)$ \\
\hline$\beta$ Blocker & $183(58 \%)$ \\
\hline ACE inhibitor & $155(49 \%)$ \\
\hline Calcium antagonist & $107(34 \%)$ \\
\hline Nitrate & $186(59 \%)$ \\
\hline Statin & $151(48 \%)$ \\
\hline \multicolumn{2}{|l|}{ LV function } \\
\hline Good (EF > 50\%) & $255(81 \%)$ \\
\hline Moderate (EF $30-50 \%$ ) & $53(17 \%)$ \\
\hline Poor (EF <35\%) & $8(2 \%)$ \\
\hline \multicolumn{2}{|l|}{ Target vessel } \\
\hline LAD & $211(49 \%)$ \\
\hline$L C x$ & $84(20 \%)$ \\
\hline RCA & $132(31 \%)$ \\
\hline \multicolumn{2}{|l|}{ ACC/AHA lesion type: } \\
\hline A & $124(29 \%)$ \\
\hline$B$ & $167(39 \%)$ \\
\hline C & $136(32 \%)$ \\
\hline \multicolumn{2}{|l|}{${ }^{*}$ Mean (SD). } \\
\hline \multicolumn{2}{|c|}{$\begin{array}{l}\text { ACC, American College of Cardiology; ACE, angiotensin converting } \\
\text { enzyme; AHA, American Heart Association; CCS, Canadian } \\
\text { Cardiovascular Society; EF, ejection fraction; LAD, left anterior } \\
\text { descending artery; LCx, left circumflex coronary artery; LV, left ventricle; } \\
\text { RCA, right coronary artery. }\end{array}$} \\
\hline
\end{tabular}

Table 2 Procedural and angiographic data of 316 patients

\begin{tabular}{ll}
\hline Interventional procedure & \\
PTCA & $40(13 \%)$ \\
Stent & $247(78 \%)$ \\
RA with or without stent & $29(9 \%)$ \\
Multivessel interventions & $101(32 \%)$ \\
Primary angiographic success & $298(94 \%)$ \\
Procedural complications & \\
Occlusive dissection & $4(1.3 \%)$ \\
Non-occlusive dissection & $24(7.6 \%)$ \\
Permanent AVC & $3(0.9 \%)$ \\
Transient AVC & $7(2.2 \%)$ \\
Side branch occlusion & $14(4.4 \%)$ \\
Thrombus & $8(2.5 \%)$ \\
Hypotension & $6(1.9 \%)$ \\
Arrhythmias & $4(1.3 \%)$ \\
Number of patients receiving abciximab & $20(6.3 \%)$ \\
\hline AVC, abrupt vessel closure; PTCA, percutaneous transluminal coronary \\
angioplasty; RA, rotational atherectomy.
\end{tabular}

positive and negative patient populations. Data were statistically analysed with the SPSS for Windows 10.0.1 (SPSS Inc, Chicago, Illinois, USA) package. A probability value of $\mathrm{p}<0.05$ was considered significant.

\section{RESULTS}

\section{Patients}

A total of 316 consecutive patients presenting with clinical symptoms of stable angina and undergoing elective PCI on native coronary vessels at our institution were included in the study. Table 1 shows the patients' demographic, clinical, and baseline angiographic characteristics.

\section{Procedural and angiographic data}

All 316 patients underwent PCI. Percutaneous transluminal coronary angioplasty alone was carried out on 40 (13\%) patients, 247 (78\%) patients had stents inserted, and 29 (9\%) underwent rotational atherectomy with or without concomitant stent insertion. Table 2 summarises procedural and angiographic data.

\section{Incidence of adverse events among patients with postprocedural cTnl increase}

In-hospital and 30 day follow up was obtained for all 316 patients. Eighteen months' follow up was obtained for 302 (96\%) patients.

\section{In-hospital events}

Sixteen $(5 \%)$ patients had adverse events in hospital: three (1\%) died, $12(3.8 \%)$ had Q wave MIs and two of these patients and one other needed inpatient CABG (1\%).

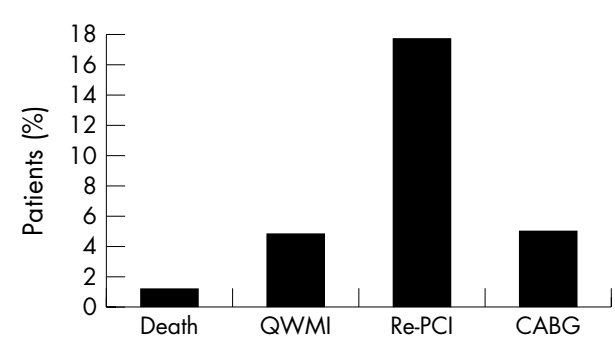

Figure 1 Cumulative adverse events at 18 months' follow up. CABG, coronary artery bypass grafting; $Q W M I, Q$ wave myocardial infarction; $\mathrm{Re}-\mathrm{PCl}$, repeat percutaneous coronary intervention. 
Events at 30 days

Twenty five (8\%) patients had an adverse event by 30 days: one $(0.3 \%)$ patient died six days after leaving hospital, six $(2 \%)$ patients had repeat PCI (four target lesion revascularisations and two de novo lesions), and two $(0.6 \%)$ further patients had CABG after leaving hospital and by 30 days.

Cumulative events at 18 months follow up Figure 1 illustrates the cumulative adverse events at 18 months' follow up.

\section{Relation between procedural angiographic complications, postprocedural cTnl increase, and outcome}

Among the total study population of 316 patients, $70(22 \%)$ patients had procedural angiographic complications (group 1). A total of $99(31 \%)$ patients had postprocedural cTnI increase (group 2) and 56 (18\%) patients had a combination of both an angiographic procedural complication and a postprocedural cTnI rise (group 3). The cumulative MACE rates were 14 of $70(20 \%)$ in group 1,41 of $99(41 \%)$ in group 2 , and 24 of $56(43 \%)$ in group 3 .

The predictive values for adverse events at follow up for each of the three groups were calculated by logistic regression. The presence of angiographic procedural complications had a PPV for adverse events of 0.3 and an NPV of 0.93 (odds ratio (OR) 4.4, 95\% confidence interval (CI) 2.2 to $8.6, \mathrm{p}=0.0003)$. The presence of a postprocedural cTnI rise had a PPV of 0.47 and a NPV of 0.96 (OR 18.9, 95\% CI 9.7 to 37, $\mathrm{p}<0.0001)$. The combination of both angiographic complication and postprocedural cTnI increase gave a PPV for adverse events of 0.69 and an NPV of 0.92 (OR 22.6, 95\% CI 2.6 to $68.6, p=0.0005)$. Figure 2 illustrates this relation.

\section{Independent predictive value of angiographic complications and postprocedural cTnl increase}

A total of 217 of $316(68.7 \%)$ patients had no postprocedural cTnI increase and $43(13.6 \%)$ of these had angiographic procedural complications. The presence of angiographic complications alone had a PPV for adverse events at follow up of 0.12 and an NPV of 0.95 (OR 2.8, 95\% CI 1.7 to 7.8 , $\mathrm{p}=0.02$ ).

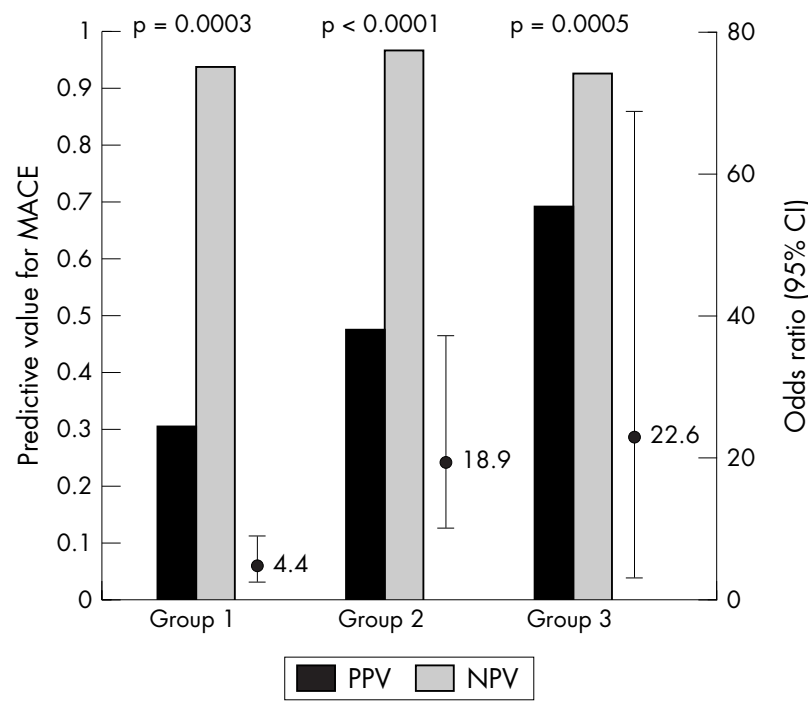

Figure 2 Predictive values for major adverse cardiac events (MACE) in the presence of angiographic complications alone (group 1), postprocedural cardiac troponin I (cTnl) increase (group 2), or both (group 3). Cl, confidence interval; NPV, negative predictive value; PPV, positive predictive value.
Table 3 Univariate analysis: relation with postprocedural cardiac troponin I increase

\begin{tabular}{llll}
\hline Variable & OR & $95 \% \mathrm{Cl}$ & $\mathbf{p ~ V a l u e ~}$ \\
\hline Age $>70$ years & 6.5 & 2.4 to 11.3 & 0.01 \\
Poor (EF <35\% ) LV function & 10.7 & 4.7 to 15.1 & 0.001 \\
ACC/AHA lesion type B & 5.4 & 2.0 to 8.6 & 0.02 \\
ACC/AHA lesion type C & 10.8 & 6.4 to 17.9 & 0.001 \\
Total balloon inflation $>180 \mathrm{~s}$ & 2.4 & 1.2 to 5.6 & 0.04 \\
Procedural complications & 23.6 & 7.9 to 29.2 & $<0.0001$ \\
\hline Cl, confidence interval; OR, odds ratio. & & \\
\end{tabular}

A total of 246 of $316(78 \%)$ of patients had no documented procedural angiographic complications and $37(12 \%)$ of them had a postprocedural cTnI rise. The PPV for adverse events at follow up of postprocedural cTnI increase alone was 0.52 and the NPV was 0.95 (OR 11.8, 95\% CI 3.4 to 18.5, p $<0.0001$ ).

\section{Relation between maximum postprocedural cTnl increase and outcome}

We retrospectively stratified patients into two groups according to the maximum postprocedural cTnI concentrations. Group A $(\mathrm{n}=186)$ comprised patients with $1-3$ times the clinical discriminant value-that is, $0.2-0.6 \mu \mathrm{g} / \mathrm{l}$ - and group B $(n=130)$ comprised patients with $>3$ times the clinical discriminant value - that is, $>0.6 \mu \mathrm{g} / \mathrm{l}$.

We found that patients in group B had an increased risk of death or Q wave MI compared with group A in hospital (3.2\% $v 1.3 \%$, respectively, $\mathrm{p}=0.0002$ ) but not at 30 days $(\mathrm{p}=0.3)$ or at 18 months' follow up $(\mathrm{p}=0.19)$. Patients in group B also had an increased risk of repeat revascularisation (repeat PCI or CABG) compared with group A $(0.95 \% v$ $0.32 \%$, respectively, $\mathrm{p}<0.01)$ in hospital but not at 30 days $(\mathrm{p}=0.64)$ or at 18 months' follow up $(\mathrm{p}=0.77)$.

Patients in group B had a significantly higher number of multivessel interventions $(\mathrm{p}<0.001)$, procedural angiographic complications (abrupt vessel closure, side branch occlusions, and occlusive dissections; $\mathrm{p}=0.02)$, and ACC/AHA type $\mathrm{B}$ or $\mathrm{C}$ lesions $(\mathrm{p}=0.007)$ than group A.

There was a significant association between postprocedural cTnI increase $>0.2 \mu \mathrm{g} / \mathrm{l}$ and the "hard" late events of cardiac death and Q wave MI (OR 3.28, 95\% CI 1.7 to $6.4, \mathrm{p}=0.01$ ).

\section{Univariate logistic regression}

Table 3 shows the relation between demographic or procedural variables and postprocedural cTnI increase within univariate analysis.

\section{Multivariate analysis}

Stepwise logistic regression analysis of the above univariate associations showed that the presence of procedural angiographic complications was the only variable to retain a significant relation with postprocedural cTnI increase $(\mathrm{p}<0.0001)$. Table 4 shows the relation with clinical outcome at 18 months.

Table 4 Multivariate analysis: relation with clinical outcome at 18 months' follow up

\begin{tabular}{llll}
\hline Variable & OR & $\mathbf{9 5 \%} \mathbf{C l}$ & $\mathbf{p ~ V a l u e ~}$ \\
\hline Diabetes & 8.3 & 2.9 to 12.6 & $<0.01$ \\
Hypercholesterolaemia & 10.8 & 4.3 to 17.9 & $<0.0001$ \\
ACC/AHA lesion type B or C & 9.5 & 2.4 to 16.2 & 0.001 \\
Procedural complications & 4.4 & 2.2 to 8.6 & 0.0003 \\
Postprocedural cTnl increase & 18.9 & 9.7 to 37 & $<0.0001$ \\
\hline CTnl, cardiac troponin I. & & & \\
\hline
\end{tabular}




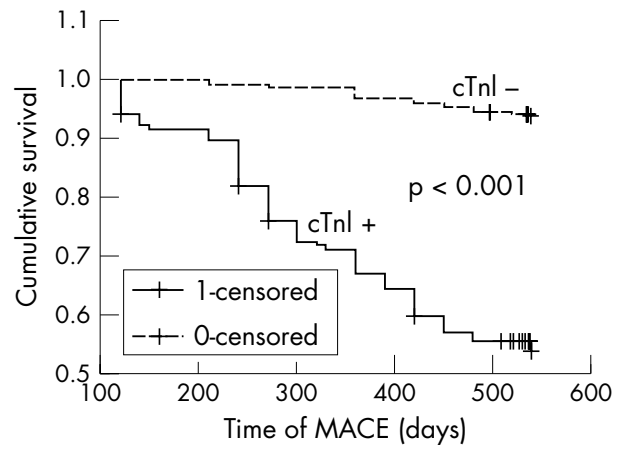

Figure 3 Kaplan-Meier curves for event-free survival according to postprocedural cTnl increase.

Event-free survival according to postprocedural cTnI Figure 3 shows the Kaplan-Meier survival curves for freedom from adverse events according to postprocedural cTnI status.

\section{DISCUSSION}

Our study was unique in that it specifically assessed clinically and "biochemically" (that is, with no preprocedural cTnI increase) stable patients. One of the key questions we set out to investigate was whether the measurement of cTnI after PCI conferred any additional diagnostic and prognostic benefit over the documentation of observed procedural angiographic complications, such as occlusive dissections and abrupt vessel closure, which are known to be associated with an increased risk of adverse outcome. ${ }^{17}{ }^{18}$ Our data showed that the predictive value of postprocedural cTnI increase was independent of, as well as additive to, that of documented procedural angiographic complications.

On univariate and multivariate regression analysis, we found that the variables with a significant association with adverse outcome were among those established factors widely used to define risk among patients undergoing PCI. Our findings were, therefore, in agreement with other reports of a significant association between post-PCI troponin increase and poor clinical outcome, with such variables as lesion complexity, increased thrombus load, and postprocedural complications (such as side branch occlusion). ${ }^{19-21}$

We found that patients with postprocedural cTnI $>0.6 \mu \mathrm{g} /$ l were more likely to have undergone multivessel interventions and to have more complex lesion morphologies and more procedural angiographic complications. The latter correlations are understandable in view of the established angiographic and procedural associations with adverse outcome, but it is more difficult to explain the apparent "all or none" effect of postprocedural cTnI increase on long term outcome. It is possible that this is a statistical observation related to sample size.

Postprocedural cTnI increase was significantly associated with the hard late events of death and Q wave MI. However, it was interesting that the greatest component of MACE in our study was repeat PCI, constituting $70 \%$ of the events by 18 months. PCI is in itself an intensely thrombogenic event, with platelet activation and aggregation, vasoconstriction, and a local and systemic thrombotic response. This acute phenomenon may be a potential substrate for subsequent fibrinous tissue formation and restenosis. It is, however, notable that not all our repeat PCIs at follow up were target lesion revascularisations, with $45 \%$ being interventions on previously untreated lesions. This may be explicable by the "inflammatory field effect" theory, whereby coronary lesions that do not appear angiographically significant at the time of the original intervention may still form the site of local inflammatory and thrombogenic activity, resulting in distal embolisation and troponin release. ${ }^{22}$ Over the follow up period, these lesions may subsequently develop into symptomatic and angiographically important entities warranting PCI.

\section{Practical implications}

Current clinical practice in most interventional centres is to measure post-PCI cardiac markers only in the presence of clinical symptoms or periprocedural complications. However, our findings would support the concept of routine measurement of troponins within 24 hours after all PCI procedures, but we accept that this requires much wider prospective validation before being universally advocated. The concept that post-PCI troponin measurement would allow the identification of potential high risk patients is attractive. Such patients may subsequently be targeted for aggressive management strategies in an attempt to positively influence their outcome: they should be more closely monitored after PCI, be subject to a more stringent follow up policy after discharge, and have a lower threshold for further investigation and treatment. A number of drugs, such as $\beta$ blockers, ${ }^{23}$ statins, $^{24}$ and glycoprotein IIb/IIIa inhibitors, ${ }^{25}{ }^{26}$ are also likely to benefit outcome in this subgroup of patients.

\section{Study limitations}

The limitations of this study are those inherent to all prospective, non-randomised registries. There was no angiographic follow up in our study and patients were subjected to further coronary angiography only on the basis of clinical symptoms, which is more representative of "real world" practice. Our study predated the results of PCI-CURE (clopidogrel in unstable angina to prevent recurrent events) ${ }^{27}$ and only patients receiving coronary stents were treated with clopidogrel. Similarly, the number of patients receiving a glycoprotein IIb/IIIa inhibitor was very small and, contrary to the more recently published supportive data, we did not give abciximab electively but as a "bail out" in complex procedures.

\section{Conclusions}

Postprocedural cTnI increased in $31 \%$ of our patient population presenting with clinically and biochemically stable coronary disease and undergoing elective PCI. In 12\% of these patients, post-PCI cTnI increase was entirely asymptomatic and occurred in the absence of angiographic procedural complications. Postprocedural cTnI increase was significantly predictive of an increased risk of adverse events, predominantly due to repeat PCI, at 18 months' follow up. The prognostic role of postprocedural cTnI increase was independent of other variables known to be associated with an increased risk of adverse outcome.

\section{Authors' affiliations}

T Nageh, M R Thomas, Department of Cardiology, King's College Hospital, London, UK

R A Sherwood, B M Harris, Department of Clinical Biochemistry, King's College Hospital

\section{REFERENCES}

1 Abdelmeguid AE, Topol EJ, Whitlow PL, et al. Significance of mild transient release of creatine kinase-MB fractions after percutaneous coronary interventions. Circulation 1996;94:1528-36.

2 Tardiff BE, Califf RM, Tcheng JE, for the IMPACT-II Investigators, et al. Clinical outcomes after detection of elevated cardiac enzymes in patients undergoing percutaneous intervention. J Am Coll Cardiol 1999;33:88-96.

3 Simoons ML, van der Brand M, Lincoff $M$, et al. Minimal myocardial damage during coronary intervention is associated with impaired outcome. Eur Heart J 1999:20:1112-9.

4 Wu AHB, Feng Y-J, Moore, et al for the American Association for Clinical Chemistry Subcommittee on cardiac troponin I standardization. Characterization of cardiac troponin subunit release into serum after acute 
myocardial infarction and comparisons of assays for troponin T and I. Clin Chem 1998;44:1198-208

5 Zimmerman J, Fromm R, Meyer D, et al. Diagnostic marker co-operative study for the diagnosis of myocardial infarction. Circulation 1999;99:1671-7.

6 Lindahl B, Venge P, Wallentin L, for the FRISC Study Group. Relation between troponin T and the risk of subsequent cardiac events in unstable coronary artery disease. Circulation 1996;93:1651-7.

7 Antman EM, Milanko J, Tanasijevic MJ, et al. Cardiac specific troponin I level to predict the risk of mortality in patients with acute coronary syndromes. N Engl J Med 1996:336:1342-9.

8 Ohman ME, Armstrong PW, Christensen RH, for the GUSTO Ila Investigators, et al. Cardiac troponin T levels for risk stratification in acute myocardial ischaemia. N Engl J Med 1996;335:1333-41.

9 Luscher MS, Thygesen K, Ravkilde J, for the TRIM Study Group, et al. Applicability of cardiac troponin T and I for early risk stratification in unstable coronary disease. Circulation 1997;96:2578-85.

10 Galvani M, Ottani F, Ferrini D, et al. Prognostic influence of elevated values of cardiac troponin I in patients with unstable angina. Circulation 1997; 95:2053-9.

11 Kizer JR, Muttraj MR, Matthei WH, et al. Role of cardiac troponin T in the long term risk stratification of patients undergoing percutaneous coronary intervention. Eur Heart J 2003;24:1314-22.

12 Fuchs S, Kornowski R, Mehran R, et al. Prognostic value of cardiac troponin I levels following catheter-based coronary interventions. Am J Cardiol 2000;85: 1077-82.

13 Matetzky S, Sharir T, Domingo M, et al. Elevated troponin I levels on admission is associated with adverse outcome of primary angioplasty in acute myocardial infarction. Circulation 2000;102:1611-6.

14 Cantor WJ, Newby LK, Christenson RH, et al. Prognostic significance of elevated troponin I after percutaneous coronary intervention. J Am Coll Cardiol 2002;39:1738-44

15 Nageh T, Sherwood RA, Harris BM, et al. Cardiac troponin I for risk stratification following percutaneous coronary intervention in acute coronary syndrome. Cathet Cardiovasc Interv 2002;55:37-42.

16 Anon. ACC/AHA/ACP-ASIM guidelines for the management of patients with chronic stable angina. J Am Coll Cardiol 1999;33:2092-197.
17 Abdelmeguid AE, Whitlow PL, Sapp SK, et al. Long term outcome of transient uncomplicated in-lab coronary artery closure. Circulation 1995;91:2733-41.

18 Piana RN, Ahmed WH, Chaitman B, on behalf of the HIRULOG Angioplasty Study Investigators, et al. Effect of transient abrupt vessel closure during otherwise successful angioplasty for unstable angina on clinical outcome at six months. J Am Coll Cardiol 1999;33:73-8.

19 Tan K, Sulke N, Taub N, et al. Clinical and lesion morphologic determinants of coronary angioplasty success and complications: current experience. J Am Coll Cardiol 1995;25:855-65.

20 Lindahl B, Diderholm E, Lagerqvist B, et al. Mechanisms behind the prognostic value of troponin $\mathrm{T}$ in unstable coronary artery disease: a FRISC II substudy. J Am Coll Cardiol 2001;38:979-86.

21 Riccardi MJ, Davidson CJ, Gubernikoff G, et al. Troponin I elevation and cardiac events after percutaneous coronary intervention. Am Heart J 2003; 145:522-8.

22 Buffon A, Biasucci LM, Liuzzo G, et al. Widespread coronary inflammation in unstable angina. N Engl J Med 2002;347:5-12.

23 Kini A, Marmur JD, Kini S, et al. Creatine kinase-MB elevation after coronary intervention correlates with diffuse atherosclerosis, and low-to-medium level elevation has a benign clinical course. J Am Coll Cardiol 1999;34:663-71.

24 Walter DH, Fichtlscherer S, Britten MB, et al. Statin therapy, inflammation and recurrent coronary events in patients following stent implantation. J Am Coll Cardiol 2001;38:2006-12.

25 The EPISTENT Investigators. Evaluation of platelet Ilb/Illa inhibitors for stenting: randomized placebo-controlled and balloon-angioplasty-controlled trial to assess safety of coronary stenting with use of platelet glycoprotein Ilb/ Illa blockade. Lancet 1998;352:87-92.

26 The ESPRIT Investigators. Novel dosing regimen of eptifibatide in planned coronary stent implantation (ESPRIT): a randomized, placebo controlled trial. Lancet 2000;356:2037-44.

27 Mehta SR, Yusuf S, Peters RJG, et al. Effects of pre-treatment with clopidogrel and aspirin followed by long-term therapy in patients undergoing percutaneous coronary interventions: the PCI-CURE study. Lancet 2001;358:527-33.

\section{IMAGES IN CARDIOLOGY}

\section{Cardiac sarcoidosis with delayed enhanced MRI}

A 40 year old Asian male collapsed while attending a wedding in India. He initially assumed that his drinks had been "spiked". He attended a hospital in India when further episodes of dizziness occurred. An ECG showed a brief run of a broad complex tachycardia. Haematology and biochemistry were normal as was the chest radiograph and a transthoracic echocardiogram. On returning to the UK he was found to have self terminating runs of polymorphic ventricular tachycardia at rates of 190 beats/min. A 12 lead ECG was normal as was a repeat echocardiogram. Coronary angiography showed no evidence of obstructive coronary artery disease.

A magnetic resonance imaging (MRI) scan was performed with a view to excluding a structural abnormality and specifically right ventricular dysplasia. Both ventricles were of normal size and function. However, on the delayed contrast enhanced images, there was hyperenhancement of the anteroseptal segments of the basal and mid thirds of the left ventricle in an epicardial distribution (panels A and B). Enlarged lymph nodes were seen in a pre-aortic
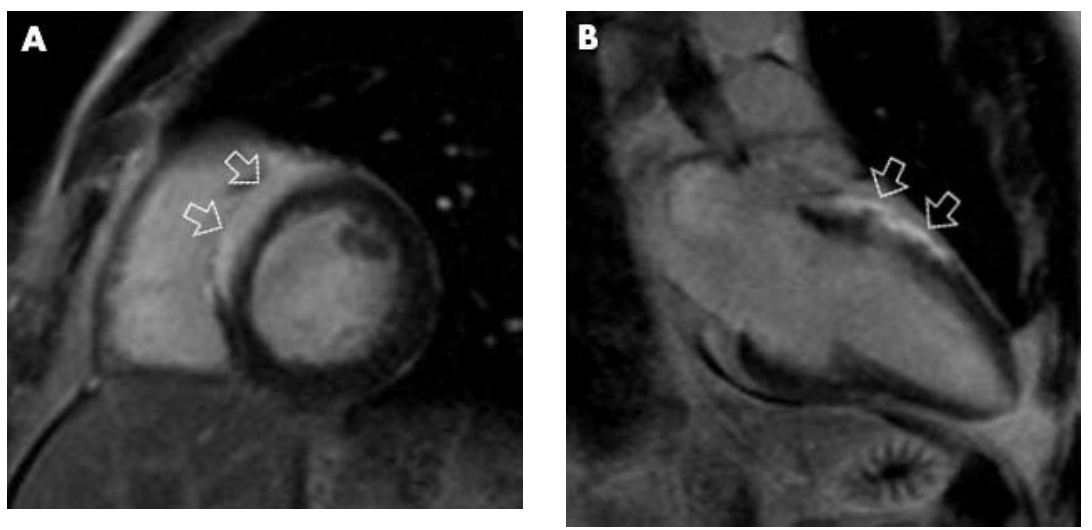

location on the $\mathrm{Tl}$ weighted images. Based on these features a provisional diagnosis of sarcoidosis was suggested. Open biopsy of a submandibular lymph node, located on ultrasound, confirmed the presence of sarcoidosis. No pulmonary manifestations of sarcoidosis were seen on the computed tomographic scan.

An automated implantable cardioverter-defibrillator was implanted. The patient remains well on sotalol, $360 \mathrm{mg} /$ day, and oral prednisolone, $20 \mathrm{mg} /$ day.

Cardiac MRI should be considered in patients with rhythm disturbance when the echocardiogram appears to show an entirely normal heart.

S W Dubrey

R Grocott-Mason

T K Mittal

simon.dubrey@thh.nhs.uk 